\title{
A Procedure to Address the Fuel Rod Failures during LB-LOCA Transient in Atucha-2 NPP
}

\author{
Martina Adorni, ${ }^{1}$ Alessandro Del Nevo, ${ }^{1}$ Francesco D'Auria, ${ }^{1}$ and Oscar Mazzantini ${ }^{2}$ \\ ${ }^{1}$ University of Pisa, GRNSPG, Via Livornese 1291, 56122 San Piero a Grado, (Pisa), Italy \\ ${ }^{2}$ Nucleoeléctrica Argentina S.A. UG-CNAII, 2806 Lima, Argentina
}

Correspondence should be addressed to Martina Adorni, m.adorni@ing.unipi.it

Received 20 September 2010; Accepted 9 December 2010

Academic Editor: Juan Ferreri

Copyright (C) 2011 Martina Adorni et al. This is an open access article distributed under the Creative Commons Attribution License, which permits unrestricted use, distribution, and reproduction in any medium, provided the original work is properly cited.

Depending on the specific event scenario and on the purpose of the analysis, the availability of calculation methods that are not implemented in the standard system thermal hydraulic codes might be required. This may imply the use of a dedicated fuel rod thermomechanical computer code. This paper provides an outline of the methodology for the analysis of the 2A LBLOCA accident in Atucha-2 NPP and describes the procedure adopted for the use of the fuel rod thermomechanical code. The methodology implies the application of best estimate thermalhydraulics, neutron physics, and fuel pin performance computer codes, with the objective to verify the compliance with the specific acceptance criteria. The fuel pin performance code is applied with the main objective to evaluate the extent of cladding failures during the transient. The procedure consists of a deterministic calculation by the fuel performance code of each individual fuel rod during its lifetime and in the subsequent LB-LOCA transient calculations. The boundary and initial conditions are provided by core physics and three-dimensional neutron kinetic coupled thermal-hydraulic system codes calculations. The procedure is completed by the sensitivity calculations and the application of the probabilistic method, which are outside the scope of the current paper.

\section{Introduction}

Loss of coolant accidents mean those postulated accidents that result from the loss of reactor coolant at a rate in excess of the capability of the reactor coolant makeup system from breaks in the reactor coolant pressure boundary, up to and including a break equivalent in size to the double-ended rupture of the largest pipe of the reactor coolant system.

A peculiarity of the Atucha-2 design is the positive void reactivity coefficient. This is a characteristic in common to other heavy-water-moderated reactors that utilize natural uranium as fuel. This implies that after a LB-LOCA event, the fission power peak at the very beginning of the transient is controlled by the void formation in the core channels, and then it is determined by the pressure wave propagation from the break. Indeed, the moderator is still liquid and flashes delayed with respect to the coolant, thus the LOCA event is also a RIA event.
Depending on the specific event scenario and on the purpose of the analysis, the availability of calculation methods that are not implemented in the standard system thermal hydraulic codes might be required, as well as burst temperature, burst strain, and flow blockage calculations. This may imply the use of a dedicated fuel rod thermomechanical computer code such as TRANSURANUS.

This paper provides an outline of the methodology for the analysis of the 2A LB-LOCA scenario in Atucha$2 \mathrm{NPP}$, focusing on the procedure adopted for the use of the fuel rod thermo-mechanical code. The methodology implies the application of best estimate thermal hydraulics, neutron physics, and fuel pin performance computer codes, with the objective to verify the compliance with the specific acceptance criteria. The fuel pin performance code is applied with the main objective to evaluate the extent of cladding failures during the transient. 
The procedure consists of a deterministic calculation by the fuel performance code of each individual fuel rod during its lifetime and in the subsequent LB-LOCA transient calculations. The boundary and initial conditions (BIC) (e.g., pin power axial profiles) are provided by core physics and three-dimensional neutron kinetic coupled thermal-hydraulic system codes calculations (see [1]). The procedure is completed by the sensitivity calculations and the application of the probabilistic method, with the aim of a better understanding of the uncertainties involved and their technological consequences on the behavior of the fuel rods, which are outside the scope of the current paper.

\section{Overview of Atucha-2 NPP}

Atucha-2 is a pressurized heavy water cooled and moderated reactor (PHWR) designed by Siemens under construction in the Republic of Argentina. The nominal electric power is $745 \mathrm{MWe}$. The reactor is equipped with a PWR-type reactor pressure vessel (RPV). The operating pressure for the moderator and the coolant is equal $(11.5 \mathrm{MPa})$ since the same fluid is simultaneously passing through the core and the moderator tank. The moderator and coolant circuits are connected through the bypass in the lower plenum and the upper plenum of the RPV. The former is constituted by two loops with U-Tubes SGs. The latter is a four-loops moderator system connecting, upstream and downstream, the moderator tank. Four horizontal U-Tubes exchangers remove the heat from the moderator system and preheat the feed water. The reactor power is generated in the core, composed of 451 fuel bundles placed in vertical fuel channels, each one containing a fuel assembly (FA) composed of 37 fuel rods. The reactor power is removed via the above-mentioned 2 hydraulic loops equipped with UTubes steam generators (SGs).

The fuel rod consists of a stack of $5.3 \mathrm{~m}$ of natural $\mathrm{UO} 2$ pellets, compensation pellets in $\mathrm{Al} 2 \mathrm{O} 3$, the supporting tube, and the compression spring. Everything is placed into a Zircaloy-4 cladding tube. Although most fission products are retained within the UO2, a fraction of the gaseous fission products is released from the pellets and accumulated in the plenum of the rod. The fuel rod cladding thickness is adequate to be "free-standing", that is, capable of withstanding external reactor pressure without collapsing onto the pellets. All fuel rods are internally prepressurized in order to reduce compressive cladding stresses and creep down due to the high coolant pressure. Helium is used as pressurizing gas to get good heat transfer from fuel to cladding.

The plant is operated with an on-power refueling that is performed by a refueling machine. After the equilibrium burnup is reached, the refueling is performed in order to move each FA just three times during its lifetime. Thus, a fresh FA is introduced into the core in a position and kept there until it reaches a certain burnup (transition burnup); then it is moved to its final location, where it is irradiated up to the discharge burnup.

\section{Atucha-2 LB-LOCA Transient}

The double ended guillotine break LOCA (DEGB LOCA or 2A LOCA) constitutes the "historical" event for the design of emergency core cooling systems (ECCS) in water-cooled reactors and is primarily adopted in vessel-equipped nuclear power plants. It is assumed that the largest pipe connected with the reactor pressure vessel (RPV) can be broken: two end breaks are generated, typically at the RPV side and at the main coolant pump (MCP) side.

In the case of Atucha-II, the history of the construction and the agreement between the regulatory authority and the utility brought to the exclusions of the 2A LOCA from the list of the design basis accidents (DBA), [2-4]. Therefore, it belongs to the category of events SBDBA (selected beyond DBA).

The analysis is performed by means of two nodalizations addressing the short tarm (10 s) and the long term (3000s) of the transient. The former is calculated with a detailed nodalization coupled with the NK ("280ch"), the latter is analyzed with a more simplified model (“60ch"), which uses the $0 \mathrm{D}-\mathrm{NK}$ and is coupled with the protection, limitation, and control systems of the NPP [4].

The BIC for the fuel pin mechanic analyses are based on the " $280 \mathrm{ch}$ " model, for the following main reasons.

(i) The higher level of detail available is more adequate for providing the boundary and the initial conditions.

(ii) Changes of the axial linear power profile, which might have a relevant impact on the results, are calculated by the $3 \mathrm{D}-\mathrm{NK}$ model, but not by the $0 \mathrm{D}$ NK model.

The analyses are limited to the first $10 \mathrm{~s}$, because the power excursion and the cladding heat up occur during this timespan. However, the long-term behavior of the fuel rods is addressed in the sensitivity analyses on the base of the results of the "60ch" nodalization. Several analyses were performed in order to investigate the worst LOCA in Atucha-II. These sensitivities addressed the break area, the break opening time, the number of boron lances available, and the break position, see [4].

\section{Description of the Transient}

Four phenomenological windows (Ph.W) are identified, based on the key phenomena and the relevant thermalhydraulic aspects occurring in Atucha-2 2A LOCA. They are (i) fission power excursion, (ii) CHF occurrence and clad temperature excursion, (iii) quenching and fuel channels refill, (iv) long-term cooling. These phases or $\mathrm{Ph} . \mathrm{W}$ are adopted instead of the classical "Blowdown", "Refill," and "Reflood" phases adopted in systematic studies of the LBLOCA in PWR [4].

4.1. Ph.W.-Fission Power Excursion (RIA). The first phenomenological window starts from the opening of the break till the time when total fuel energy achieves $90 \%$ of the value 
attained when power equals the decay value (this is to ensure that the RIA part of the transient is terminated and to include in this period possible damage mechanisms originated by energy deposition in the fuel, see Figure 1). The duration of the first $\mathrm{Ph} . \mathrm{W}$ is of the order of one second. The propagation of the depressurization wave that originated at the break and the consequent occurrence of the fission power peak are the characterizing phenomena. Start of the CHF condition occurs in this period.

4.2. Ph.W.-CHF Occurrence and Clad Temperature Excursion. The second window lasts from the end of the previous window till (roughly) the time of actuation of accumulators. The duration of the second window is of the order of ten seconds. The widespread of the CHF condition and the rod surface temperature excursion, including the occurrence of the peak cladding temperature and the related turnaround caused by liquid flashing and by flow reversal in the core, are the characterizing phenomena. Containment pressurization also occurs during this $\mathrm{Ph} . \mathrm{W}$.

4.3. Ph.W.-Quenching and Fuel Channels Refill. The third window ends when liquid level (mixture) fills all the core channels. At the beginning of the Ph.W. early quench occurs noticeably before the actuation of emergency systems and is caused by flow reversal in the core and flashing. The ECCS intervention keeps the clad temperature down and is necessary for refilling of the fuel channels. The duration of the third phenomenological window is of the order of a few minutes. In this period, equalization of pressures between containment and primary circuit occurs.

4.4. Ph.W.-Long-Term Cooling (LTC). The fourth Ph.W implies the continued containment sump recirculation operation and ensures the "post-LOCA long-term" core cooling. The behavior of the sump (liquid level and temperature, other than debris effect), the performance of the SIP, and the level distribution (and stabilization) in the primary loop constitute the characterizing thermal-hydraulic phenomena.

\section{Fuel Safety Criteria}

An overview of fuel safety criteria relevant for the fuel performance of CNA-2 NPP is briefly quoted in this section. Reference is given to the document issued by UNIPI and related to "proposal for performing the Atucha-2 accident analyses for licensing purposes", [2] according to the international practice (see also [5]).

Specific acceptance criteria for design basis accidents for CNA-2 NPP are reported in Table 1. Criteria that are addressed by TRANSURANUS code are highlighted in red, notwithstanding the classification of SBDBA of the LBLOCA in Atucha-II.

In addition to these, in the current approach, the extent of fuel failure of $10 \%$ has been considered as reference (according to German licensing regulations) [6].
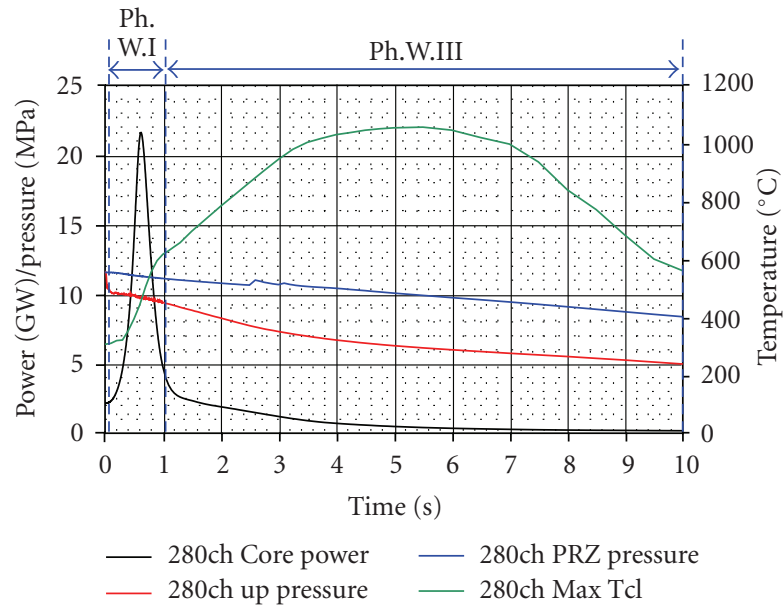

FIgURE 1: Power, pressure, and maximum cladding temperature, "280ch" 3D-NK.

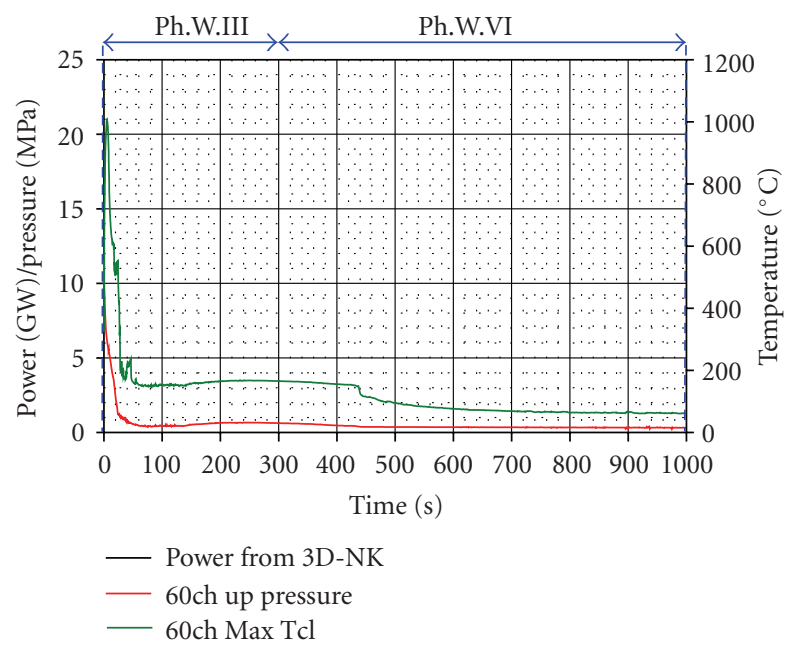

FIGURE 2: Power, pressure, and maximum cladding temperature, all the transient, "60ch" 0D-NK.

\section{Core Status}

The Atucha-2 reactor has on-power re-fueling. There are three refueling paths, as summarized hereafter and they depend upon burnup.

Path 1. Fresh fuel enters zone 6, stays until a transition burnup, moves to 5 , stays until it reaches the exit burnup, and leaves the core.

Path 2. Fresh fuel enters zone 2, stays until a transition burnup, moves to 4 , stays until it reaches the exit burnup and leaves the core.

Path 3. fresh fuel enters zone 3, stays until a transition burnup, moves to 1 , stays until it reaches the exit burnup and leaves the core. 
TABLE 1: Specific acceptance criteria for design basis accidents [2].

\begin{tabular}{ll}
\hline Safety parameter & Criterion for the DBA \\
\hline Fuel temperature & $T_{\max }<T_{\text {melt }}$ for $90 \%$ of pellet cross-section at hot spot \\
Fuel enthalpy (for RIA) & Average fuel hot-spot enthalpy $<180$ cal/g for irradiated fuel \\
Heat transfer cladding/coolant & Departure from nucleate boiling (DNB) is admissible, except for the cases listed below under* \\
Cladding temperature & $T_{\text {clad }}<1200^{\circ} \mathrm{C}$ except for the cases listed below* when $T_{\text {clad }}<650{ }^{\circ} \mathrm{C}$ \\
Cladding integrity (for LOCA) & Limited loss of integrity admissible maximum local oxidation $<17 \%$ \\
Core-wide oxidation (for LOCA) & Maximum hydrogen generation $\mathrm{H}_{2}<1 \%$ of $\mathrm{H}_{2 \text { TOTAL }}$ \\
Operation of PZR safety valves & Challenge admissible \\
RCS pressure & ASME code level C service limit $\left(p<1.2 p_{\text {design }}\right)$ \\
Secondary side pressure & ASME Code Level B Service Limit $\left(p<1.2 p_{\text {design }}\right)$ \\
Containment pressure & Maximum pressure $<$ design pressure \\
Permissible dose & Calculated doses below limits $10 \mathrm{CFR} 50.670 .25 \mathrm{~Sv}$ total effective dose equivalent 0.05 Sv total \\
\hline
\end{tabular}

* Examples of events with potential primary medium release outside the containment are (i) SG tube or moderator cooler tube rupture (with emergency mode), (ii) Long-term loss of main heat sink with SG or moderator cooler tube leakage (equal to operational leakages) (iii) Main steam line rupture outside containment with SG or moderator tube leakage (iv) Break of an instrument line in the annulus.

The fuel can be considered divided in two groups: the fuel that has not been subjected to shuffling and fuel that has been moved after reaching the transition burnup of the belonging zone.

Fuel burn-up zones are represented in Figure 3. All the fuel channels are identified according to RELAP5-3D model. Different colors represent different refueling paths. The calculations are performed at the equilibrium burnup distribution for the reference core for each fuel assembly.

The 451 fuel assemblies are represented one by one in the neutronic model whereas the thermal hydraulic model represents the 451 core channels with 280 "pipe" components. The association between neutronic and thermal hydraulic conditions for each fuel rod is carried out according to Figure 3. Each rectangle corresponds to a channel in the reactor. Internally, the number represents the identification of the RELAP5-3D model: they are consecutive numbers from 250 to 529 . Each channel is, then, identified with 2 coordinates according to the neutronic schematization.

Linear heat rate is provided by the 3D-NK model (451 values) assuming the 37 rods of each fuel assembly contribute with the same power. The temperatures and pressures $(280$ values) are the ones of the corresponding hydraulic channel, see Figure 3 for correspondence.

\section{TRANSURANUS Overview}

TRANSURANUS is a computer program for the thermal and mechanical analysis of fuel rods in nuclear reactors (see [7-10]). The TRANSURANUS code consists of a clearly defined mechanical-mathematical framework into which physical models can easily be incorporated. The mechanicalmathematical concept consists of a superposition of a onedimensional radial and axial description (the so-called quasitwo-dimensional or 1.5-D model). The code was specifically designed for the analysis of a single cylindrical rod.
On the basis of the defined boundary conditions, the code calculates the temperature distribution and the fission gas release inside the fuel rod, the corresponding inner pressure, the $\mathrm{ZrO} 2$ thickness growth, the equivalent oxidation, and the plastic deformation of the cladding. Fuel rod burst is checked through appropriate failure criteria [5].

TRANSURANUS code can also be applied in design basis accident (DBA) analyses, including LOCA and RIA, to complement system level simulations and to verify the fuel-specific safety acceptance criteria on the basis of detailed thermo-mechanical computations.

The LOCA-specific models available in the code are validated up to value of cladding temperature equal to $1200^{\circ} \mathrm{C}$.

\section{TRANSURANUS Loca Specific Failure Criteria}

The option Loss Of Coolant Accident (LOCA) analysis in TRANSURANUS code activates specific models either for fresh fuel rods or fuel rods with specific burnup (which include an analysis of the normal operation prior to transient). This latter method provides the unique possibility of the consistent simulation of fuel rod performance under normal operation and accident conditions. The model and material property can be defined specifically for LOCA analysis. The transition between normal and LOCA-specific models is at the user-defined time set in the LOCA input block (tloca).

Three different failure criteria are available in TRANSURANUS code, specific for LOCA conditions [7].

The first cladding failure criterion is based on stress assessment, that is, the comparison of the calculated tangential stress with a distinct failure threshold (overstress criterion). The failure threshold of the cladding alloy is defined as a function of the actual temperature and oxidation level in the material property function. 

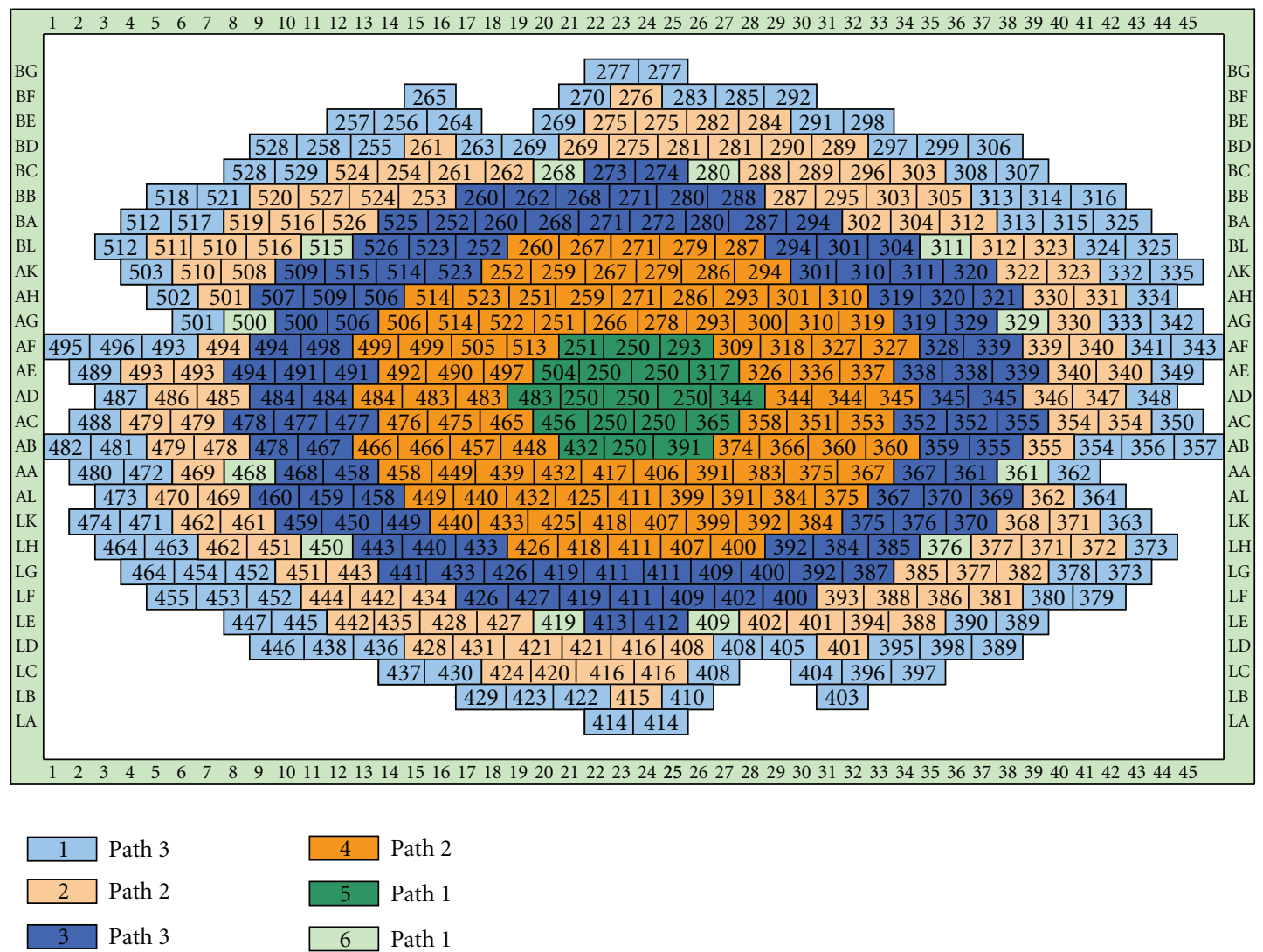

FIGURE 3: Fuel burnup zones and RELAP5-3D fuel channels distribution, [1].

The second failure principle is a simple "plastic instability criterion" based on the simultaneous assessment of the effective true strain and the strain rate. When both the strain and the strain rate exceed the threshold values of 0.025 and $100 \mathrm{1} / \mathrm{h}$, respectively, (values set by the user in the input) $[7,11]$, the cladding is assumed to be ruptured. These parameters are introduced in the model as input parameters. This criterion is applied concurrently to an "overstrain criterion" (third out of three criteria) that is, if plastic instability is not indicated, then the overstrain criterion is checked automatically. This criterion limits the true tangential plastic strain to the maximum of $50 \%$. These criteria were introduced particularly for LOCA analyses, see [8], due to the significant uncertainty of the stress computation at large cladding deformation.

\section{Base Irradiation}

The base irradiation is the time period from the insertion of the fresh fuel rod into the core, up to the time at which the equilibrium burnup of the reference core is reached. Distinctions between rods that are and are not subject to shuffling are separately discussed in the following sections.

Base irradiation calculations are performed in order to reach the equilibrium burnup of the reference core.
9.1. No Shuffling. The base irradiation of the fuel rods that are not subjected to shuffling, (corresponding to the fuel zones 2, 3, and 6 of Figure 3 ) is performed by imposing the axial linear heat rate, the fast flux, the axial temperature, and the pressure histories, which are specific for each fuel rod. This allows them to reach the corresponding burnup conditions as evaluated for the equilibrium core, used for the FSAR preparation. 2A-LOCA transient starts after the base irradiation, from the "rod-specific" linear heat rate, temperature, pressure, and fast flux of the steady state according to the RELAP5-3D calculation.

9.2. Shuffling. The fuel assemblies subjected to shuffling correspond to the fuel zones 1,4 , and 5 of Figure 3. For those assemblies, the base irradiation can be divided into three zones, according to the representation of Figure 4:

(a) the zone before shuffling (up to t transition burnup),

(b) the shuffling itself (between $\mathrm{t}$ transition burnup to t10), and

(c) the zone after the shuffling (between $\mathrm{t} 10$ to $\mathrm{t}$ LOCA).

9.2.1. Zone before Shuffling. Three refueling paths with a different transition burnup values are envisaged in Atucha-2, they correspond to the zones 1,4 , and 5 . 


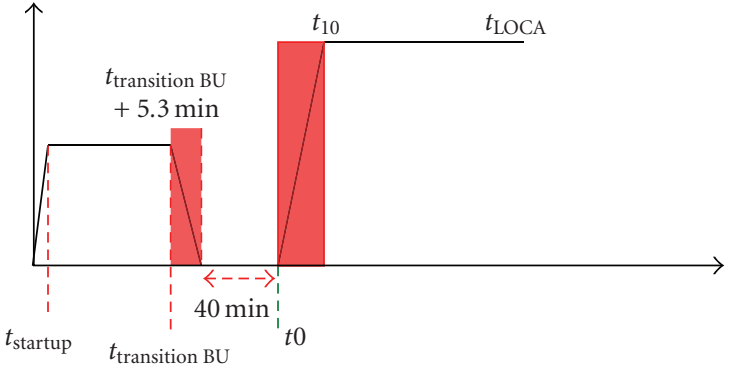

Figure 4: Timing of refueling.

Temperature distribution and pressure from the steadystate values of the RELAP5-3D, specific for each rod, are modeled as boundary conditions for the base irradiation of the rods belonging to these zones. Three values of linear heat rate, corresponding to the maximum of each zone of Figure 3, are modeled.

9.2.2. Refueling Zone. The fuel element withdrawal and insertion velocities are modeled using the value of $1 \mathrm{~m} / \mathrm{min}$. The fuel residence time outside the core is set to $40 \mathrm{~min}$ according to the information available from NA-SA,. Additional assumptions are made for extraction and insertion of the fuel rods, as described in the following part.

Case of Rod Extraction. The refueling modeling is performed in "steps". Considering that the fuel model is divided into 10 axial segments and the length of each segment is $0.53 \mathrm{~m}$, the time elapsed for taking out of the core each segment, considering that the withdrawal and insertion velocities of $1 \mathrm{~m} / \mathrm{min}$, is $0.53 \mathrm{~min}$. Between two values, the linear heat rate is linearly interpolated.

Relative axial power history during refueling in function of relative axial position is reported in Figure 5 for each time step of $0.53 \mathrm{~min}$. The minimum linear heat rate is equal to the decay heat, as shown in the figure.

During refueling, the pressure remains constant at the steady-state value. The axial cladding temperature profile changes in step from the actual value to the temperature calculated at decay power conditions with the coolant temperature of the core inlet at full-power steady state.

Case of Rod Insertion. The pressure is constant at the value of the reference steady state. The temperature as well as the linear heat rate axial distribution is accounted for in the calculation using the algorithm reported here and the reference steady-state values. The linear heat rate corresponds to the decay conditions.

This formulation is applied for both linear heat rate and temperature.

The axial power history as a function of time for the 10 axial positions is reported in Figure 6. This figure represents the relative axial power as a function of time; each dot represents a step of $0.53 \mathrm{~min}$. In this sample case, three axial positions of the rods (from the bottom to position 3 ) exhibit an increase of relative axial power followed by a decrease.

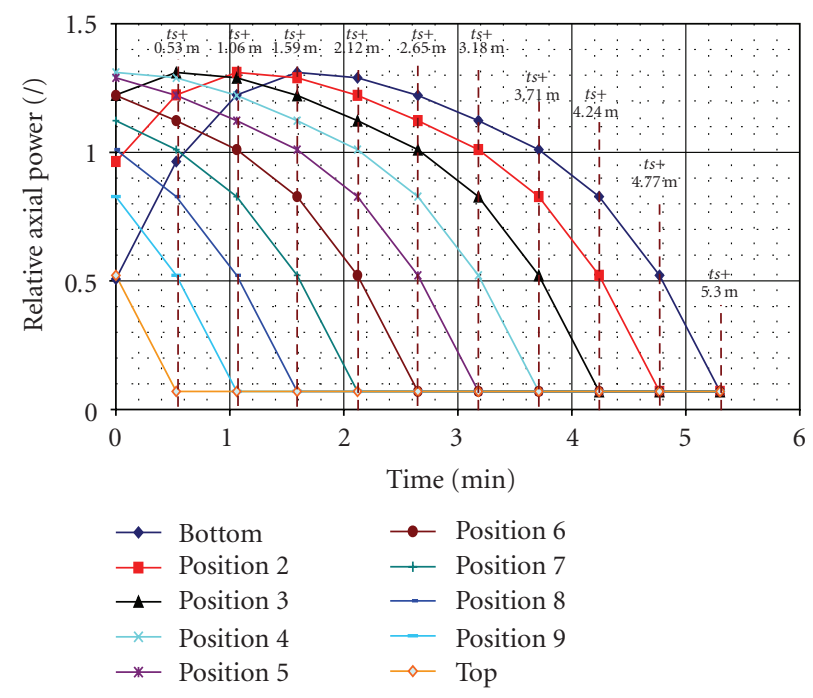

FIGURE 5: Relative axial power history during refueling as a function of time for different axial position fuel rod extraction.

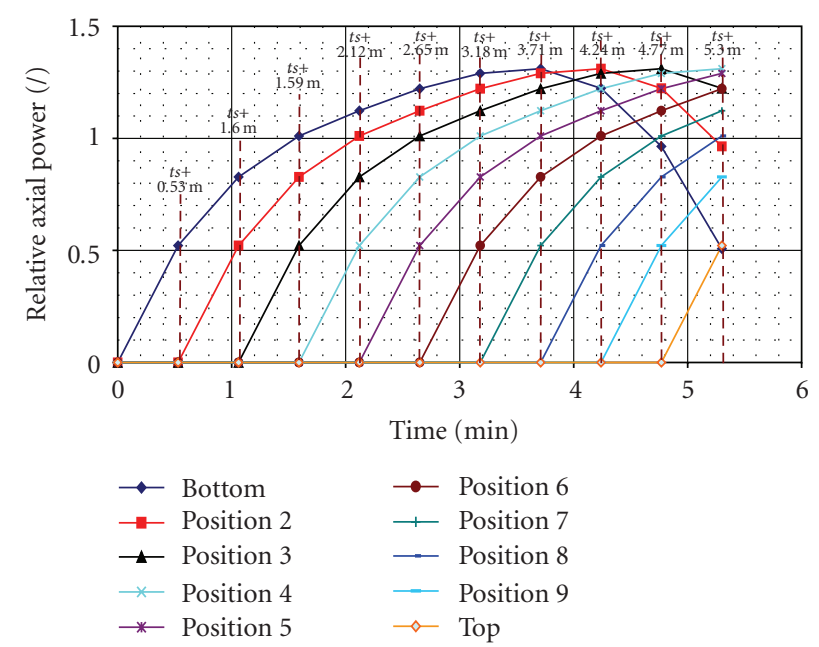

FIGURE 6: Relative axial power history during refueling as a function of time for different axial position, fuel rod insertion.

Indeed, the lower part of the rod passes from a region at low power to a region at a higher power and finally it returns in a lower power zone. The position of the rod corresponding to the maximum axial power value exhibits only a decrease of linear heat rate.

During refueling, pressure remains constant. The axial cladding temperature profile changes in step from the actual value to the temperature calculated at decay power conditions with the coolant temperature of the core inlet at full-power steady state.

9.3. Zone after Shuffling up to Equilibrium Burnup Distribution of the Reference Core. Base irradiation after refueling is performed considering constant linear heat rate, cladding temperature distribution and pressure up to the equilibrium burnup distribution, for the reference core. 


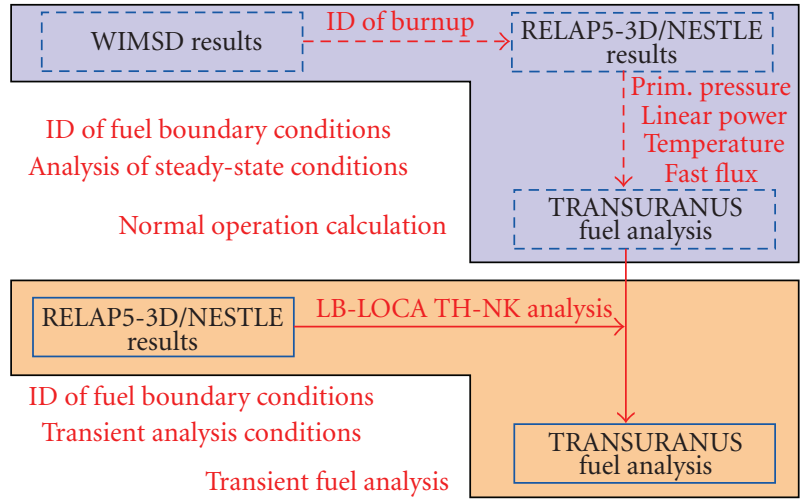

FIGURE 7: Outline of code interactions.

\section{2A-LOCA Transient}

2A-LOCA transient calculations start after the base irradiation, at the time when each rod reaches the equilibrium burnup of the reference core.

Two additional analyses of the core are performed to complement the deterministic calculation, assuming all the rods at burnup equal to $0 \mathrm{MWd} / \mathrm{kgU}$ (fresh fuel) and $8 \mathrm{MWd} / \mathrm{kgU}$ (maximum burnup allowed). The base irradiation of these series of runs is performed with the following assumptions:

(i) axial shape of linear heat rate and fast flux constant in time at the value of the steady-state neutron kinetic calculations (specific for each rod);

(ii) axial shape of temperature constant in time at the value of the steady-state thermal hydraulic calculations (specific for each rod);

(iii) pressure of the channel at the value of the steady state thermal hydraulic calculations (specific for each rod).

The results of these two additional analyses are not reported in the current paper.

\section{Assembly Radial Peaking Factors}

The radial peaking factors for the power are accounted for in the analyses. The ring-to-ring-power distribution was calculated by NA-SA using WIMSD code ([13]).

\section{MATLAB Transfer Program}

The boundary conditions selected for the fuel analysis to be implemented in the "MACRO" part of the input are:

(i) linear heat rate,

(ii) rod surface temperature,

(iii) pressure, and

(iv) fast neutron flux.

Figure 7 illustrates the codes' interactions.
RELAP5-3D, internally coupled with NESTLE code for the neutron kinetic analysis, provides

(i) power in [W], for 451 hydraulic channels, 10 axial positions;

(ii) cladding temperature in $[\mathrm{K}]$, for 280 hydraulic channels, 10 axial positions;

(iii) pressure in $[\mathrm{Pa}]$; for 280 hydraulic channels, 1 axial position;

(iv) fast neutron flux in $\left[\mathrm{n} / \mathrm{cm}^{2} \mathrm{~s}\right]$, for 451 neutronic channels, 10 axial positions.

Calculations are performed through a program written in MATLAB language, a brief outline is reported below.

The program is written to run with MATLAB, and is prepared in order to perform automatically the analysis of all the fuel rods at the selected burnup and considering the refueling strategy. An outline of the program block is reported in Figure 8.

The program, written in blocks to facilitate the review, needs as input

(i) the output of the preliminary calculations performed by RELAP5-3D/NESTLE code,

(ii) the "DATA BLOCK" part of the TRANSURANUS input,

(iii) the TRANSURANUS code and annex files, and

(iv) some "input files" which allow the program to perform the runs automatically.

A transferring map provides then the correspondences among the real NPP configuration (451 fuel assemblies with 37 fuel pin each) and the $\mathrm{TH}$ and the NK representations (280 TH channels and $451 \times 10$ neutron kinetic nodes).

The data are then transferred in the TRANSURANUS input models. Finally, the program automatically runs the code and stores the results for all the rods.

The program is able to cope with different radial peaking factors different burnup and refueling strategies.

\section{Results}

The fuel analysis is carried out using the version " $\mathrm{v} 1 \mathrm{~m} 1 \mathrm{j} 08$ " of TRANSURANUS code. It consists of the simulation of the 451 rods, each one representing 1 out of 451 fuel assemblies.

The results discussed hereafter are based on one selected 2A LOCA reported in [4]. First the rod analysis is focused on the average behavior of the fuel in the assembly. Then, the application of assembly radial peaking factors is addressed to distinguish, as far as possible, the different behavior of the rods in assembly.

The LB-LOCA transient starts at the time where the equilibrium burnup of the reference core is reached for each rod. Four rods representing four fuel assemblies are calculated to fail.

The list of the failed assemblies is reported in Table 2. The table highlights the time of failure, the burn-up value 


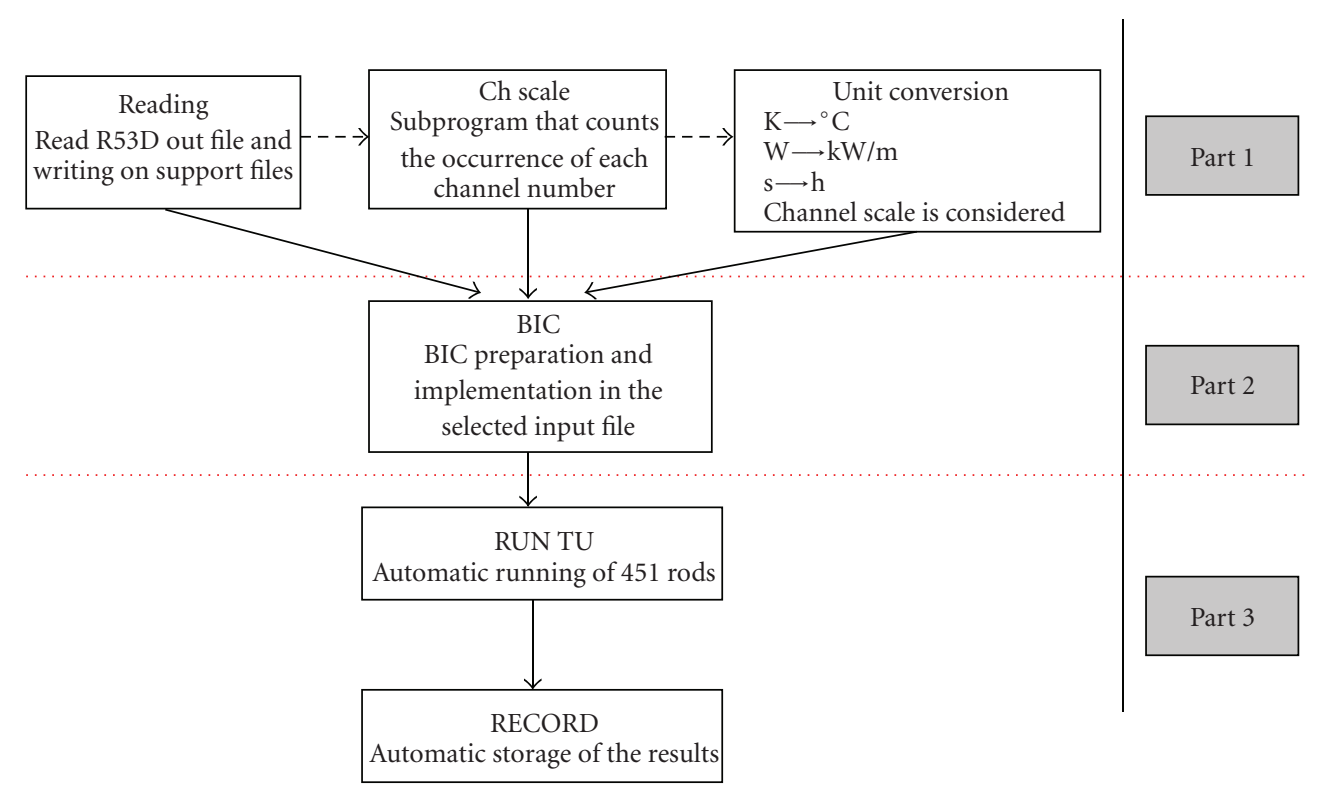

FIGURE 8: Outline of program blocks.

TABLE 2: Failed rods, case of equilibrium burnup of the reference core.

\begin{tabular}{lccccc}
\hline Assembly no. & Time[s] ${ }^{*}$ & BU/BUmax & PCT/PCTmax & LHR/LHRmax & Zone \\
\hline 467_AB11 & 2.75 & 0.572 & 1 & 0.960 & Note \\
267_AK22 & 5.13 & 0.931 & 0.960 & 0.914 & DTmin \\
327_AF33 & 3.02 & 0.528 & 0.971 & 1 & 4 \\
267_BL21 & 5.32 & 0.870 & 0.960 & 0.914 & 4 \\
\hline
\end{tabular}

* From the beginning of LB-LOCA, from the end of base irradiation.

at the beginning of the LOCA, the ratio between PCT and LHR and their maximum values, and the zone of the core where the rods are placed and the predicted cause of failure. Figure 9 reports in black the failed fuel assemblies modeled by the average representative fuel rod. One out of 451 code calculations does not reach convergence (i.e., the time step required is less than the minimum time step). This fuel rod (assembly 467_AB11) is assumed to fail because it exhibits the maximum PCT after about $2.75 \mathrm{sec}$ from the beginning of the LOCA.

Among the other three failures:

(i) assembly 267_AK22 is predicted failed just after the cladding temperature peak during the quench phase of the LOCA (i.e., after about $5 \mathrm{sec}$ from the beginning of the LOCA);

(ii) the assembly 327_AF33 is predicted failed during the cladding heatup (i.e., after about $3 \mathrm{sec}$ from the beginning of the LOCA);

(iii) the assembly 267_BL21 fails for plastic instability just after the cladding temperature peak during the quench phase of the LOCA (i.e., after about $5 \mathrm{sec}$ from the beginning of the LOCA).
Figure 10 reports the time trend of the pellet outer radius, cladding inner radius and the contact pressure between fuel and cladding, and the gap and coolant pressure of the equivalent rod corresponding to the assembly 267_BL21. The fuel and the cladding at the beginning of the LOCA transient are in contact, and the contact pressure shows a maximum value of about $6 \mathrm{MPa}$. The equivalent stress and the burst stress together with the creep strain as a function of the time are depicted in Figure 11. The burst stress is a decreasing function of temperature (burst stress is lower at higher temperature). The rod fails to plastic instability. This means that the sum of the effective plastic strain and effective creep strain and the strain rate exceed the limits that are set through the input deck for strain and strain rate. Figure 12 reports the fuel outer radius and cladding inner radius as function of time for the rod 267_BL21 for the overall irradiation.

Figure 13 reports the outer cladding radius as a function of burnup for all the rods. The higher values are predicted at lower burnup. The maximum value is exhibited by the rod corresponding to the assembly 267_BL21 (not reported in the figure) that is predicted failed for plastic instability. Notwithstanding the low burnup, the rod no. 272_BA24 exhibits the highest value of cladding strain and high value 


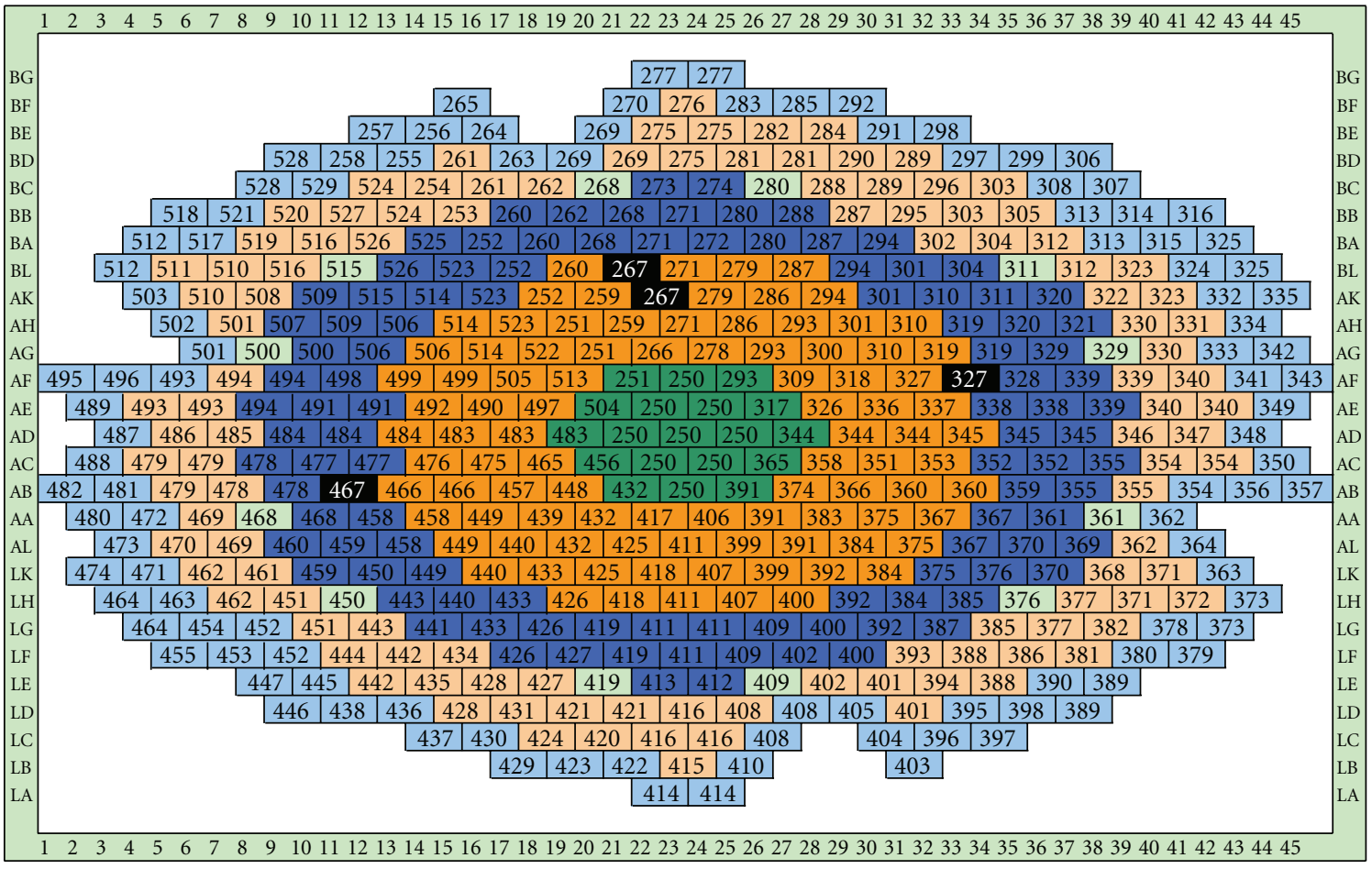

FIGURE 9: Failure predicted by TRANSURANUS code, case equilibrium burnup of the reference core accounting for shuffling.

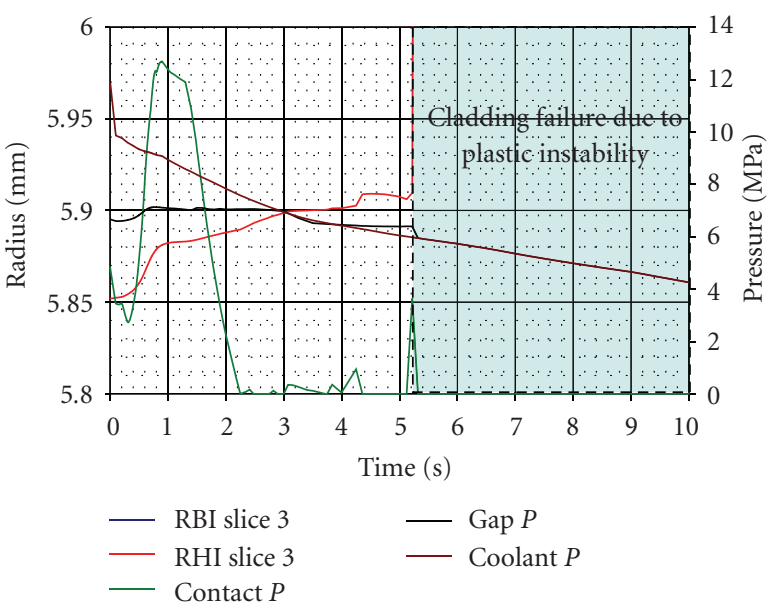

FIGURE 10: Outer fuel and inner cladding radius, contact, gap, and coolant pressure as a function of time, Rod 267_BL21 slice \#3.

because it has the maximum cladding temperature and linear heat rate. Another important parameter, affecting fuel cladding behavior under transients and postulated accidents, is the internal rod pressure. As it can be seen from Figure 14, the higher the pressure, the higher the resulting cladding outer radius. Also in this figure, the rod no. 272_BA24 exhibits the highest pressure and the highest cladding outer radius.

Tables 3 and 4 summarize the outcomes of the analyses providing the average values of the selected quantities calculated per each burnup zone and the maximum value.

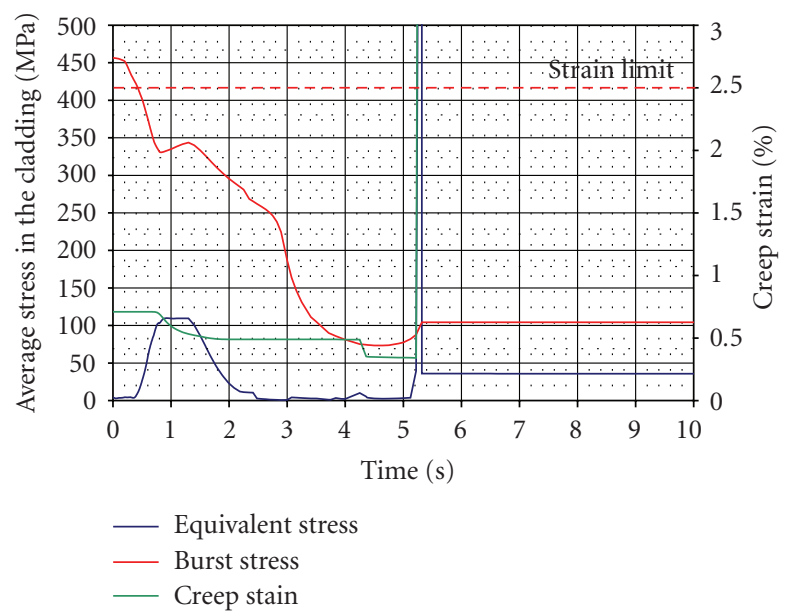

FIGURE 11: Equivalent and burst stress and creep strain as a function of time, Rod 267_BL21 slice \#3.

13.1. Effect of the Assembly Radial Peaking Factors. The evaluation of the number of the pin failures in the core is performed considering the FA radial peaking factors [12]. It is assumed that the power distribution inside the fuel assembly is characterized by an azimuthal symmetry. Each ring of Figure 7 is characterized by a specific linear power proportional to the NESTLE steady-state and transient calculations. The other parameters assumed as boundary and initial conditions of the fuel pin mechanics analyses are those utilized for the reference calculation. The results are reported below separately for each ring. 


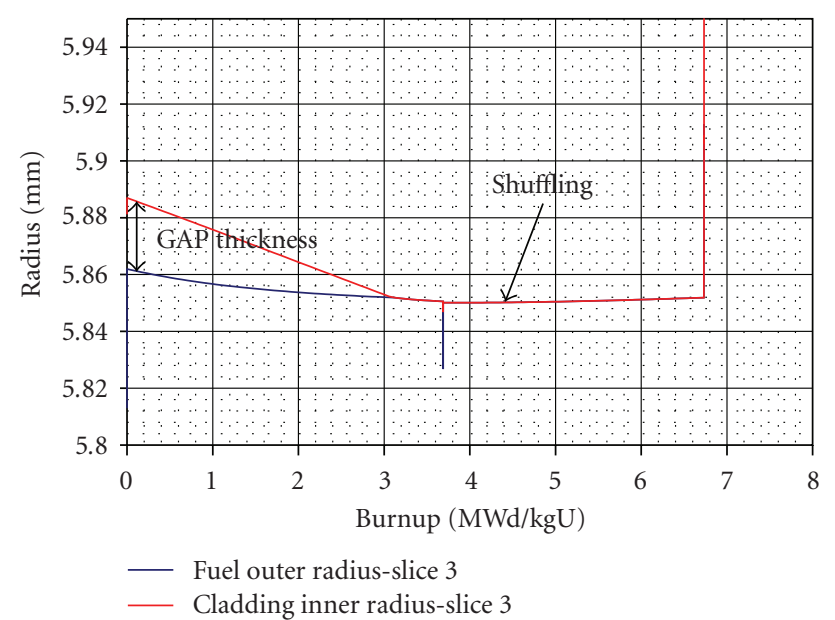

FIGURE 12: Outer cladding radius as a function of burnup, reference case and all the rods.

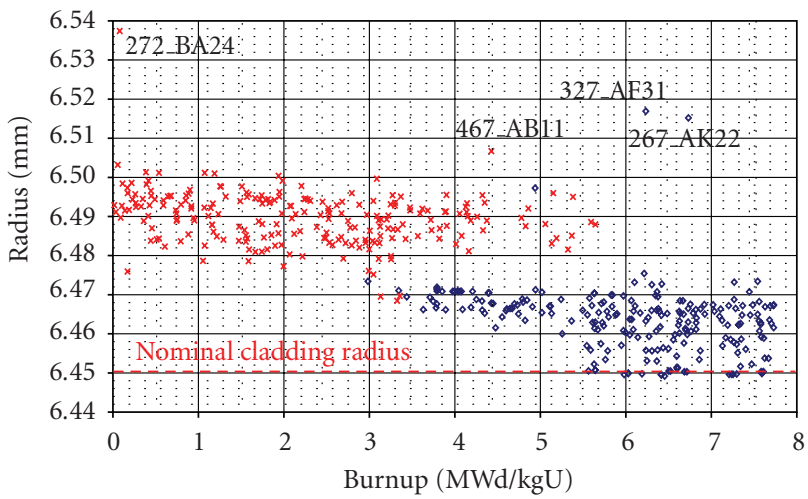

$\diamond$ Zones 1,4 , and 5

$\times$ Zones 2,3 , and 6

*267_BL21 $7.58 \mathrm{~mm}$ not reported

FIGURE 13: Cladding outer radius as a function of gap pressure.

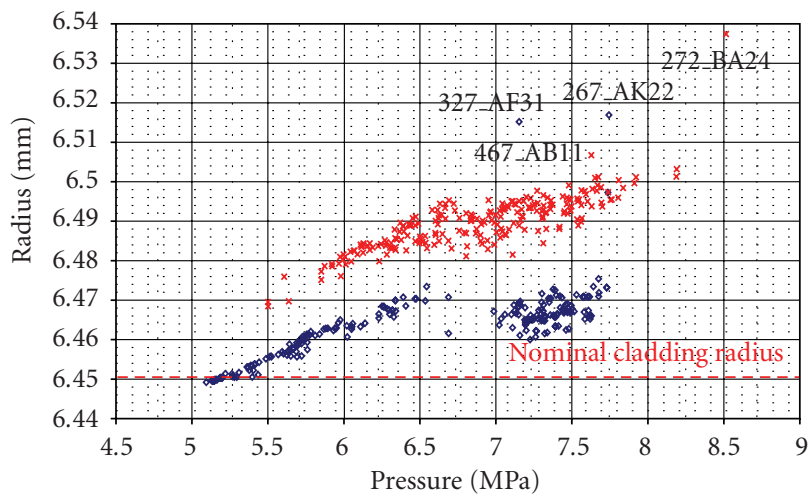

$\diamond$ Zones 1,4 , and 5

$\times$ Zones 2,3 , and 6

*267_BL21 $7.58 \mathrm{~mm}$ not reported

Figure 14: Fuel outer radius and cladding inner radius as a function of burnup; rod 267_BL21, and overall irradiation.
TABLE 3: Main outcomes from the analysis: zones 1, 4, and 5.

\begin{tabular}{lcccc}
\hline Parameter & $\begin{array}{c}\text { Average } \\
\text { Zone 1 }\end{array}$ & $\begin{array}{c}\text { Average } \\
\text { Zone 4 }\end{array}$ & $\begin{array}{c}\text { Average } \\
\text { Zone 5 }\end{array}$ & Max \\
\hline T cladding $\left[{ }^{\circ} \mathrm{C}\right]$ & 579.7 & 841.0 & 823.5 & 959.5 \\
T Fuel cl $\left[{ }^{\circ} \mathrm{C}\right]$ & 760.7 & 1595.8 & 1625.4 & 1860.7 \\
Enthalpy $[\mathrm{cal} / \mathrm{g}]$ & 45.0 & 83.7 & 83.7 & 99.1 \\
Gap P [MPa] & 5.7 & 7.3 & 7.5 & 7.74 \\
FGR [\%] & 1.9 & 0.2 & 0.2 & 3.17 \\
Gap width $[\mu \mathrm{m}]$ & 58.8 & $47.2^{*}$ & 50.6 & 60.0 \\
Cladding outer & 6.46 & 6.48 & 6.47 & 7.6 \\
radius [mm] & 0.45 & 0.42 & 0.40 & 0.58 \\
ECR [\%] & 3.25 & 3.06 & 2.92 & 4.24 \\
Ox Thick. $[\mu \mathrm{m}]$ &
\end{tabular}

${ }^{*}$ Rod no. 267_BL21 excluded from the calculation of the average and maximum.

TABLE 4: Main outcomes from the analysis: zones 2, 3, and 6.

\begin{tabular}{lcccc}
\hline Parameter & $\begin{array}{c}\text { Average } \\
\text { zone 2 }\end{array}$ & $\begin{array}{c}\text { Average } \\
\text { zone 3 }\end{array}$ & $\begin{array}{c}\text { Average } \\
\text { zone 6 }\end{array}$ & Max \\
\hline T cladding $\left[{ }^{\circ} \mathrm{C}\right]$ & 779.8 & 855.8 & 850.5 & 988.4 \\
T Fuel cl $\left[{ }^{\circ} \mathrm{C}\right.$ ] & 1315.6 & 1652.2 & 1681.9 & 2054.9 \\
Enthalpy [cal/g] & 74.4 & 88.6 & 89.9 & 106.5 \\
Gap P [MPa] & 6.5 & 7.3 & 7.3 & 8.5 \\
FGR [\%] & 0.01 & 0.08 & 0.01 & 0.84 \\
Gap width [ $\mu \mathrm{m}]$ & 63.6 & 61.4 & 62.0 & 69.0 \\
Cladding outer & 6.49 & 6.49 & 6.49 & 6.54 \\
radius [mm] & & & & \\
ECR [\%] & 0.33 & 0.34 & 0.32 & 0.41 \\
Ox Thick. $[\mu \mathrm{m}]$ & 2.37 & 2.48 & 2.36 & 3.03 \\
\hline
\end{tabular}

The applied radial peaking factors for the linear heat rate, with reference to Figure 7, are

(i) 3rd ring containing 18 rods, linear heat rate multiplication factor: 1.1065

(ii) 2 nd ring containing 12 rods, linear heat rate multiplication factor: 0.9296;

(iii) 1st ring containing 6 rods, linear heat rate multiplication factor: 0.8508 ;

(iv) central rod, linear heat rate multiplication factor: 0.8222 .

The percentage of failed rods considering the whole core, composed by the 16687 fuel rods (assembled in 451 fuel assemblies), and distinguishing the different assembly radial power peaking factors resulted in $1.13 \%$, for the considered scenario.

The failed rods are grouped per ring as follow:

(i) 3rd ring: 126 failed rods out of 8118 ,

(ii) 2nd ring 48 failed rods out of 5412,

(iii) 1st ring 12 failed rods out of 2706 , and

(iv) central rod 2 failed rods out of 451. 
126 out of 188 failed rods are predicted to lose their structural integrity during the heat up, when the power peak is already passed and the temperature is approaching the maximum. The ratio between the maximum local temperature for the considered rod and the maximum temperature calculated during LOCA for all the core for these rods is always above 0.9 . The remaining 62 rods, are predicted to fail during the quenching of the cladding.

\section{Conclusions}

The analysis of the 2A LB-LOCA in Atucha-2 NPP implies the application of best estimate thermalhydraulics, neutron physics, and fuel pin performance computer codes, with the objective to verify the compliance with the specific acceptance criteria. This paper provides a description of the methodology and the procedure adopted for the analysis and the interactions among the codes for evaluating the behavior fuel rods with respect to their acceptance criteria. The analysis is performed by means of the thermo-mechanical code TRANSURANUS.

The procedure consists of calculating the initial conditions of each rod of the core for a specific core configuration, and, then, the behavior on transient of the fuel pin on the base of a high detailed three-dimensional neutron kinetic coupled thermal-hydraulic system code (RELAP53D) calculation. The consideration of the assembly radial peaking factor allows the distinguishing of the different behaviors of the rods belonging to the same assembly. The procedure is completed by the sensitivity calculations and the application of a probabilistic method for the evaluation of the uncertainty not discussed in the present paper. The analyses of the TRANSURANUS results demonstrate that the percentage of fuel rods failures, for the selected 2A LBLOCA transient, is well below $10 \%$.

Finally, the outcomes of the calculations will be also used to evaluate the radioactive release following LB-LOCA accident.

\section{Nomenclature}

BDBA: Beyond design basis scenario

BEPU: Best estimate plus uncertainties

BU: Burnup (in general in $[\mathrm{MWd} / \mathrm{kGU}]$ )

CHF: Critical heat flux

DBA: Design basis accident

DEGB: Double ended guillotine break

ECC: $\quad$ Emergency core cooling

ECR: $\quad$ Equivalent cladding reacted

FA: $\quad$ Fuel assembly

FC: $\quad$ Fuel channel

FGR: Fission gas released

GRNSPG: Gruppo di ricerca Nucleare San Piero a Grado

ITU: Institute for Transuranium Elements

LB-LOCA: Large break loss of coolant accident

LHR: Linear heat rate

LOCA: Loss of coolant accident

MATLAB: Matrix laboratory

NA-SA: Nucleoélectrica argentina S.A.
NK: $\quad$ Neutron kinetic

NPP: $\quad$ Nuclear power plant

PCI: $\quad$ Pellet cladding interaction

PCT: $\quad$ Peak cladding temperature

PCMI: Pellet cladding mechanical interaction

Ph.W: $\quad$ Phenomenological window

PHWR: Pressurized heavy water reactor

PRZ: $\quad$ Pressurizer

PWR: $\quad$ Pressurized water reactor

PSAR: $\quad$ Preliminary safety analysis report

RELAP5-3D: Reactor excursion and leak analysis program 3D

RIA: $\quad$ Reactivity initiated accident

RPV: $\quad$ Reactor pressure vessel

SG: $\quad$ Steam generator

SIP: $\quad$ Safety injection pump

TH: Thermalhydraulics

UNIPI: $\quad$ University of Pisa

3D: $\quad$ Three-dimensional.

\section{References}

[1] C. Parisi, O. Mazzantini, F. D’Auria, and K. N. Ivanov, "RELAP5-3D(C) 3D-NK coupled TH analyses of the Atucha2 PHWR," in Proceedings of the International Conference on the Physics of Reactors: Nuclear Power: A Sustainable Resource (PHYSOR '08 ), Interlaken, Switzerland, September 2008.

[2] UNIPI, "A Proposal for Performing the Atucha II Accident Analyses for licensing purposes," Contract NA-SA UNIPI 02Atucha II, Rev. 3, August 2008.

[3] NASA-ARN, "Protocol of Common Understanding on the Basic Licensing Concept and some safety subjects," 8./9.11.1977.

[4] F. D'Auria et al., "DEGB LBLOCA $(2 \times 100 \%$ Break in CL $)$ in Atucha-2 NPP," Contract NA-SA UNIPI 01-Atucha II, DIMNP NT 628(08)—rev.1, Pisa, Italy, March 2008.

[5] "Fuel Safety Criteria Technical Review," OECD/CSNI/PWG2 Task Force on Fuel Safety Criteria, NEA/CSNI/R(99)25, July 2000.

[6] "Fuel cladding failure criteria," European Commission, EUR 19256EN, September 1999.

[7] K. Lassmann, A. Schubert, P. Van Uffelen, C. Gyory, and J. van de Laar, Transuranus Handbook version v1m1j06, JRC, EC, ITU, 2006.

[8] P. Van Uffelen, C. Gyori, A. Schubert, J. van de Laar, Z. Hózer, and G. Spykman, "Extending the application range of a fuel performance code from normal operating to design basis accident conditions," Journal of Nuclear Materials, vol. 383, no. 1-2, pp. 137-143, 2008.

[9] K. Lassmann, "TRANSURANUS: a fuel rod analysis code ready for use," Journal of Nuclear Materials, vol. 188, no. C, pp. 295-302, 1992.

[10] P. Van Uffelen, "Modelling of Nuclear Fuel Behaviour," Report EUR 22321 EN, JRC, 2006, European Commission.

[11] "Atucha-2 FSAR," Final Safey Analysis Report, 2007.

[12] O. Mazzantini, "Information requested through DIT (dated 20MAR2008)," Contract NA-SA UNIPI 02-Atucha II, NASAUNIPI 06, July 2008.

[13] WIMSD code, "NEA Data Bank Documentation". 

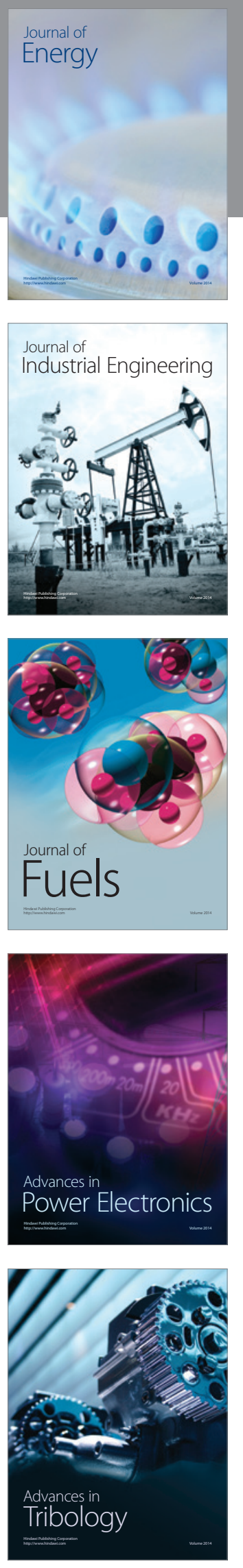
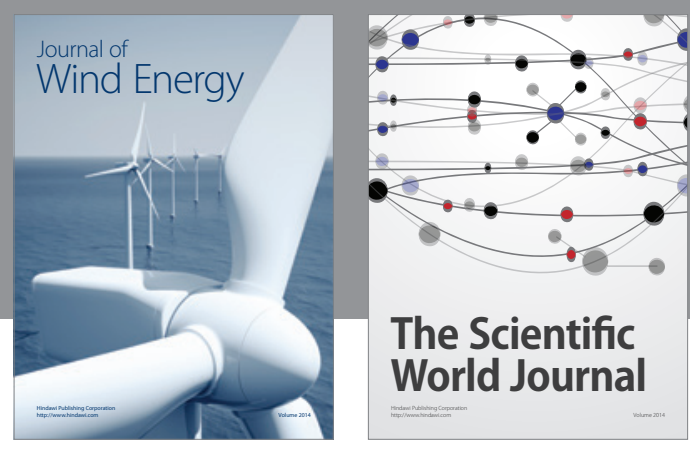

The Scientific World Journal

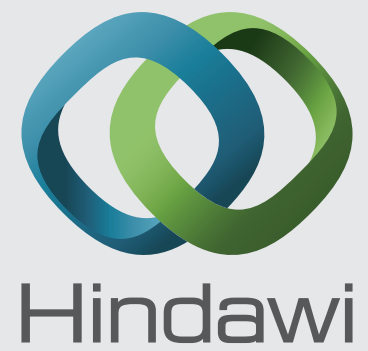

Submit your manuscripts at http://www.hindawi.com
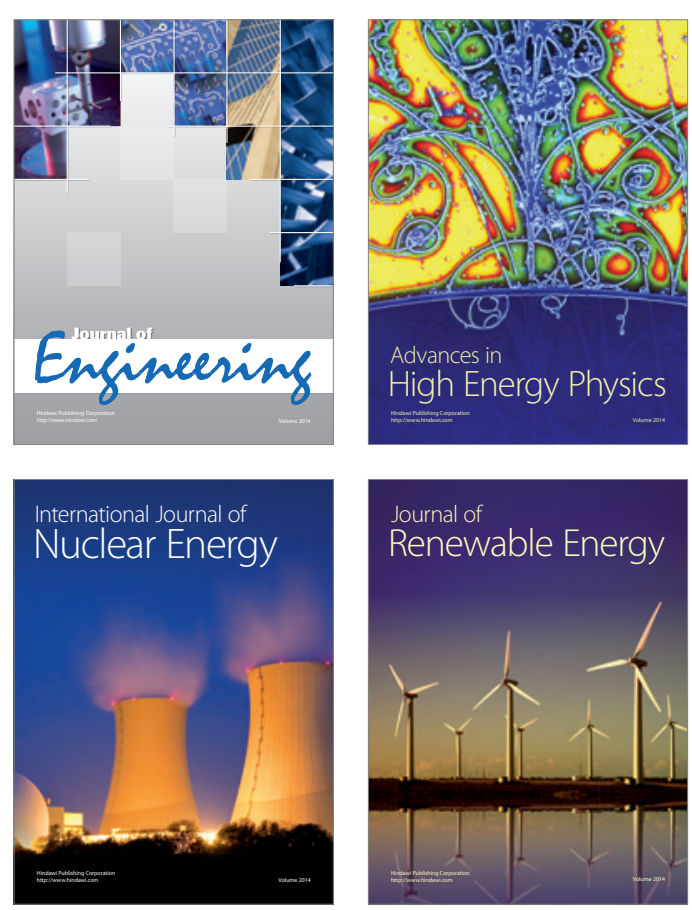

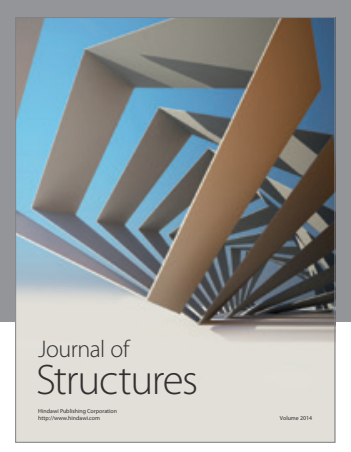

Rotating
Mechinery
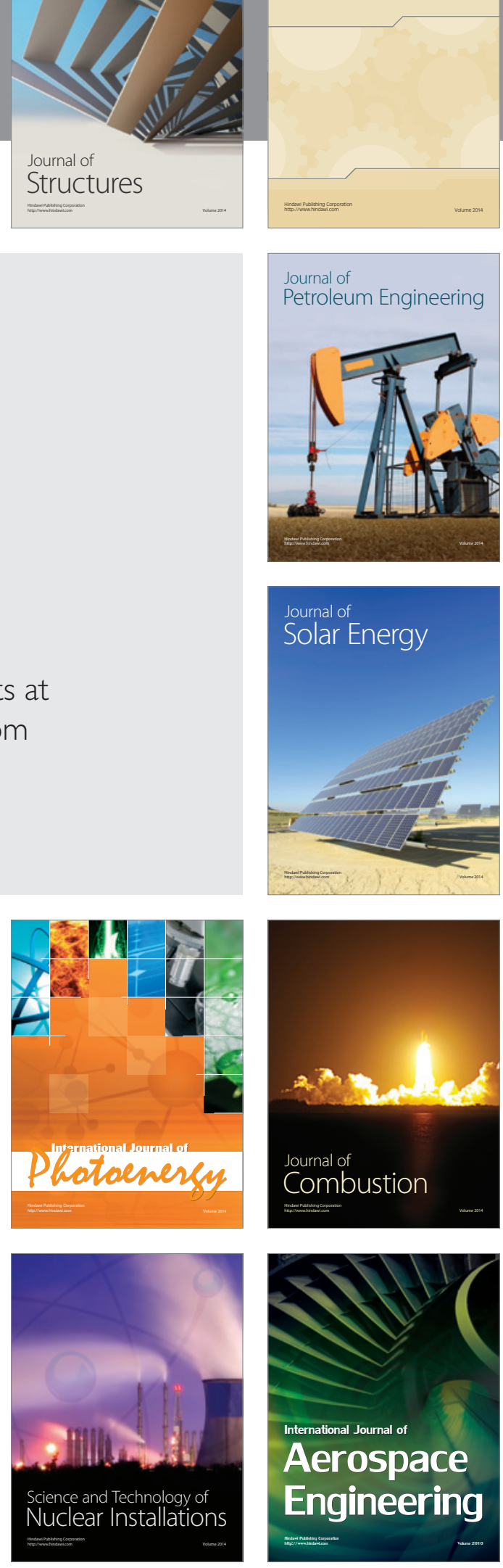